\title{
A VERY STRAIGHT AND COLLIMATED OUTFLOW IN THE CORE OF OMC-1
}

\author{
J. SCHMID-BURGK, R. GÜSTEN, R. MAUERSBERGER, A. SCHULZ and T. L. WILSON \\ Max-Planck-Institut für Radioastronomie, Auf dem Hügel 69, D-5900 Bonn 1, RFA
}

\section{Summary}

We have recently discovered a large-scale $\left(200^{\prime \prime}\right)$ outflow system in the core of OMC-1 (fig. 1), centered about 100" South of IRc2 and extending over some 120" (red lobe) resp. $60^{\prime \prime}$ (blue) along a position angle of $-31^{\circ}$ (Schmid-Burgk et al. 1990). The blue lobe which might actually protrude into the HII region M42 is poorly defined in CO 2-1, but the red lobe reveals a number of remarkable properties which we summarize here:

1. The outflow is very straight and smooth. Over the full length of $120^{\prime \prime}$, the center of any cross scan deviates by not more than about $1^{\prime \prime}$ from a straight line. This line passes to within $2^{\prime \prime}$ the peak of the submm source FIR4 of OMC-1 (Mezger, Wink and Zylka 1990) and the mm continuum peak CS3 (Mundy et al. 1986); it also cuts across the red and blue SiO-outflow lobes recently discovered some 5-10" to either side of FIR4 (Ziurys, Wilson and Mauersberger 1990). It thus seems that the "base" of our large-scale $\mathrm{CO}$ jet can be seen as well.

2. The collimation (length to width $\geq 15$ ) is among the highest ones known. The diameters of the most redshifted velocity components are unresolved at $230 \mathrm{GHz}$, i.e. smaller than $4^{\prime \prime}$, over the full extent of the jet.

3. Where measurable, the lobe diameter depends on velocity in a systematic way at any given distance from the origin of the flow, the most redshifted velocities being the narrowest. All velocity components are centered on the same axis. The fastest motions thus are embedded in a wider envelope of slow flow. The total $\mathrm{V}_{L S R}$ range of the redshifted wing extends over $\approx 17 \mathrm{~km} \mathrm{~s}^{-1}$.

4. For any velocity, the signal strength increases with distance from FIR4, culminating $60^{\prime \prime}$ away before a steep decline sets in. The decline zone is unresolved by our $12.5^{\prime \prime}$ beam. At $\approx 65^{\prime \prime}$, the first stage of the outflow must thus come to an abrupt halt. Further on, the flow continues to be seen at lower levels, eventually building up to a second peak near 110". As before, buildup of signal strength proceeds sequentially from low velocities to high. This results in a linear increase of flow speeds with distance, hence an acceleration approaching exponential behaviour in time. The characteristic time scale is 17000 years, to be reduced by projection factors which may be considerable.

5. Where measurable, the flow's brightness temperature is invariably at a constant value near $45 \mathrm{~K}$, similar to that of the ambient gas $(40 \ldots 70 \mathrm{~K})$. No significant heating or cooling seems connected with the flow, not even at the position of abrupt decline (the "obstacle"). 


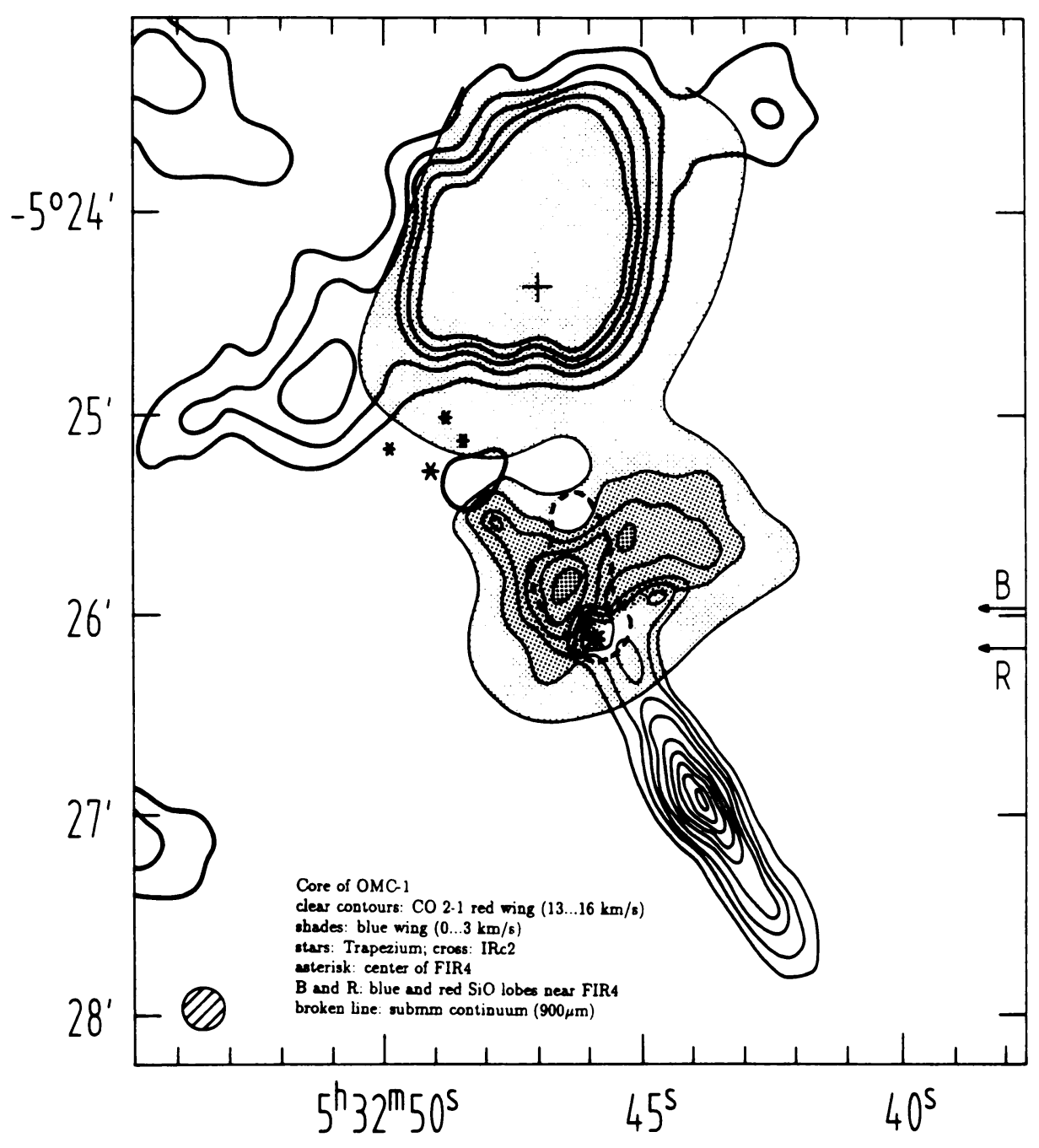

Fig.1. The newly discovered outflow and its surroundings in OMC-1. The four stars are the Trapezium, the cross is IRc2, and FIR4, the suspected origin of the bipolar flow system, is indicated by the asterisk some $80^{\prime \prime}$ South-West of the Trapezium stars. 
6. The flow is optically thick in CO 2-1 everywhere except at the very tip. The densities must thus be at least a few $10^{5} \mathrm{~cm}^{-3}$, and the total mass loss rate could reach several $10^{-4} M_{\odot}$ per year. At $\approx 75^{\prime \prime}$ from FIR4, the ${ }^{12} \mathrm{CO} /{ }^{13} \mathrm{CO} 2-1$ intensity ratio is 24 , indicating $\mathrm{n}_{H_{2}} \approx 3 \cdot 10^{5} \mathrm{~cm}^{-3}$. Densities probably exceed the ambient gas values.

7. A "halo" of what nearly seems like countercurrents, of blueshift a few $\mathrm{km} \mathrm{s}^{-1}$ relative to the ambient molecular gas, can be seen to a distance of $10^{\prime \prime}$ from the axis of the redhifted jet. This might be ambient gas somewhat compressed (but not heated) by the action of the flow, or turbulent matter transported from the vicinity of FIR4, or else rotation of the jet material around its length axis.

8. Near FIR4 itself, a structure perpendicular to the outflow axis is clearly seen in the blue wing of $\mathrm{CO} 2-1$ at $\mathrm{V}_{L S R} \approx 0$ to $5 \mathrm{~km} \mathrm{~s}^{-1}$ to the West and, much less clearly, in the red to the East of the lobes. This invites speculations on a dynamical structure from which the flow might emanate.

\section{References}

Mezger P. G., Wink J. E., Zylka R. 1990 Astron. Astrophys. 228, 95.

Mundy L. G., Scoville N. Z., Baath L. B., Masson C. R., Woody D. P. 1986 Astrophys. J. Lett. 304, L51. Schmid-Burgk J., Güsten R., Mauersberger R., Schulz A., Wilson T. L. 1990 to appear in Astrophys. J.

Letters.

Ziurys L. M., Wilson T. L., Mauersberger R. 1990 Astrophys. J. Letters 356, L25. 